\title{
Barreras al emprendimiento hotelero: un análisis sectorial
}

\author{
Barriers to hotel entrepreneurship: a sector analysis
}

\section{Rubén Lado-Sestayo}

Universidade da Coruña, Departamento de Economía Financiera y Contabilidad, Dpto. Economía Financeira e Contabilidade, Facultade de Economía e Empresa (UDC), Campus de Elviña s/n, 15071 A Coruña, España, ruben.lado.sestayo@udc.es

\section{Milagros Vivel-Búa}

Universidad de Santiago de Compostela, Departamento de Economía Financiera y Contabilidad, 15782 Santiago de Compostela, España, mila.vivel@usc.es

\section{Luis Otero-González}

Departamento de Economía Financiera y Contabilidad de la Universidad de Santiago de Compostela, 15782 Santiago de Compostela España, luis.otero@usc.es

\section{Isabel Neira-Gómez}

Departamento de Economía Cuantitativa de la Universidad de Santiago de Compostela, 15782 Santiago de Compostela España, isabel.neira@usc.es

\section{Resumen}

Este trabajo analiza el impacto de las barreras de entrada existentes en una localización turística sobre la intensidad emprendedora en el sector hotelero español durante el período 2005-2011. En concreto, se estudia una muestra formada por 8,992 establecimientos hoteleros y se utilizan cuatro tipos de modelos, a saber, Poisson, Binomial Negativa, modelo Truncado y regresión Logística. Las variables de estudio se refieren al nivel de concentración empresarial, al tamaño de mercado, la rentabilidad, la ocupación, la inversión, la fuerza laboral y los cierres. Los principales resultados indican la importancia del nivel de concentración competitiva como factor que influye negativamente en la creación empresarial en esta industria. Al mismo tiempo, también debe subrayarse el efecto positivo ejercido por el tamaño de mercado.

Palabras clave: Emprendimiento, barreras de entrada, hotel, España, punto turístico.

\begin{abstract}
This paper analyzes the impact of the entry barriers existing in a tourist location on the intensity in the entrepreneurial sector hotel Spanish for the period 2005-2011. In particular, we study a sample of 8,992 hotels by means of four models, namely, Poisson, Negative Binomial, Truncated Models and Logistic Regression. Variables used are related to the level of business concentration, the market size, profitability, employment, investment, employees and closures. Main results point the importance of the level of market concentration as a competitive factor influencing negatively on new ventures. Also, it should also be stressed the positive effect exerted by market size.
\end{abstract}

Keywords: Entrepreneurship, entry barriers, hotel sector, Spain, tourist destination.

(a)

\section{Introducción}

La superación de la crisis económica actual supone el establecimiento y consolidación de un tejido productivo dinámico y eficiente donde, entre otros factores, el emprendimiento se alza como una herramienta importante, sobre todo, debido a su impacto sobre el crecimiento económico (Acs, Braunerhjelm, Audretsch, \& Carlsson 2009). De hecho, el emprendimiento actúa como un elemento dinamizador de la industria favoreciendo el nivel de competitividad en los distintos sectores (Porter 2008). Como consecuencia, uno de los objetivos perseguidos por las instituciones gubernamentales es favorecer la creación de nuevas empresas.

En las últimas décadas han surgido diversas investigaciones focalizadas en analizar la figura del emprendedor en cuanto a sus características personales, sociales y/o económicas (Verheul, Wennekers, Audretsch \& Thurik 2002; Li 2008). Sin embargo, uno de los aspectos menos tratados en la literatura previa es la caracterización de estas iniciativas empresariales. En este sentido, es importante destacar que la actividad emprendedora puede responder a una oportunidad, a una necesidad, o a una mezcla de ambas motivaciones. Consecuentemente, ello puede condicionar el establecimiento de objetivos, por ejemplo, priorizando la supervivencia a la generación de un gran crecimiento en un horizonte temporal relativamente breve, su capacidad de adaptación al entorno e incluso su papel en el mercado.

Centrándonos en el sector hotelero, la actividad emprendedora ha demostrado ser una importante vehículo para su desarrollo con efectos sobre su nivel de competitividad (Ateljevic \& Page 2009; Mottiar \& Ryan 2007; Santos-Góes \& Vianna-Brugni 2014). El incremento en la tasa de emprendimiento hotelero favorece la aparición de nuevas empresas, lo que aumenta el nivel de competitividad de los distintos puntos turísticos contribuyendo al desarrollo sectorial (Kalnins \& Chung 2004; Santos, Ferreira \& Costa 2014). A su vez, cabe destacar que una de las particularidades del sector hotelero es la importancia de la localización porque, por ejemplo, el producto ofrecido por un establecimiento solo puede ser consumido en el lugar donde éste se encuentra ubicado. De hecho, Russell \& Faulkner (2004) subrayan que el emprendimiento en el ámbito del turismo está influido en gran medida por el estado de desarrollo de la localización. Aquellas localizaciones que presentan mayores obstáculos a la creación empresarial, presentarán, al menos a priori, menores tasas de 
emprendimiento frente a otras más proclives a la entrada de competidores lo cual favorece la creación de nuevas empresas (Gémar-Castillo \& Jiménez-Quintero 2013). Por tanto, uno de los principales aspectos a considerar en el estudio acerca del impacto que la localización ejerce sobre la actividad emprendedora hotelera es la potencial existencia de barreras al emprendimiento.

El objetivo de este trabajo es analizar qué factores actúan como barrera de entrada en la creación de nuevas empresas hoteleras y determinar cuál es su impacto durante el período 2005-2011. Si bien la influencia de la localización sobre el emprendimiento ha sido analizada en trabajos centrados en otros sectores (Stam 2007; Parwada 2008), todavía no existen investigaciones empíricas en este sector que, en el caso de España, tiene un papel relevante en la economía nacional. Además, España ocupa una posición importante en el contexto turístico mundial, por lo cual una investigación de este tipo puede generar resultados relevantes y/o extrapolables a otras economías. Por ejemplo, España es líder europeo y ocupa la segunda posición a nivel mundial en la obtención de ingresos por turismo internacional en el año 2012. Además, es la cuarta economía mundial en la recepción de turistas, igualada con China y sólo superada por Francia y EE.UU.

Este trabajo se organiza en seis apartados. Tras esta introducción, el segundo apartado recoge la revisión de la literatura y, a continuación, el tercero presenta las hipótesis de trabajo y variables de análisis. El cuarto apartado se refiere al estudio empírico realizado, exponiendo la metodología aplicada y los resultados obtenidos. El trabajo concluye con un quinto epígrafe que sintetiza las conclusiones y un sexto que recopila las referencias bibliográficas utilizadas.

\section{Revisión de la literatura}

Las investigaciones sobre la actividad emprendedora han centrado su atención en analizar sus determinantes a nivel individuo, pudiendo distinguirse tres grupos de trabajos. En primer lugar, se encuentran aquellos centrados en el análisis de las características individuales tales como los rasgos psicológicos del emprendedor (Sánchez Lanero \& Yurrebaso 2005), su género (Verheul et al., 2002) y/o su aversión al riesgo (Stewart \& Roth 2001). En segundo lugar, están los estudios que evalúan los determinantes sociales como, por ejemplo, el papel del capital social o la cultura emprendedora (Thurik \& Dejardin 2011). Finalmente, existe un tercer grupo de trabajos que evalúan la influencia del entorno económico, destacando aquellos que relacionan la actividad emprendedora con la tasa de desempleo (Justo \& Díaz, 2012) o con el tamaño del mercado (Figueroa-Armijos, Dabson \& Jonhson 2012; Monterrubio \& Mendoza-Ontiveros 2014).

En el caso del emprendimiento en la industria turística, cabe señalar que no existe abundante literatura, sobre todo, al ponerla en relación con aquella disponible para ámbitos sectoriales y considerar su importancia en la economía de algunos países, como es el caso de España. Además, estos trabajos se centran en evaluar los determinantes focalizándose en el individuo y su comportamiento (Jaafar, Abdul-Aziz, Maideen \& Mohd 2011; Li 2008). Por ejemplo,
Ramos-Rodríguez, Medina-Garrido \& Ruiz-Navarro (2012) estudia los determinantes del emprendimiento turístico incipiente a partir de datos del Global Entrepreneurship Monitor en el año 2008. Al mismo tiempo, también existen algunos trabajos que analizan el emprendimiento corporativo, esto es, qué factores presentes en una organización favorecen una actitud emprendedora en sus trabajadores (Li, Ching-Yick \& Zhao 2009; Martínez-López \& Vargas-Sánchez 2013; Carlsson, Braunerhjelm, McKelvey, Olofsson, Persson \& Ylinenpa 2013).

Frente a estas dos unidades de análisis, individuo y empresa, no existe todavía literatura que se centre en evaluar el entorno donde operan estas nuevas iniciativas empresariales resultantes del proceso emprendedor, si bien autores como Stenholm, Acs \& Wuebker (2013) pusieron de relieve su importancia en el dinamismo y eficiencia sectorial. Por ejemplo, respecto al entorno legal, se ha demostrado que, en ocasiones, el efecto de la legislación sobre la creación empresarial guarda relación con la influencia que ejercen las empresas ya instaladas sobre las decisiones políticas con el objetivo de preservar su posición en el mercado (Rodríguez \& Murdy 2006; De la Cruz 2003). De hecho, algunos autores apuntan que esta posición de ventaja por parte de las empresas instaladas se ve reforzada en zonas de baja intensidad competitiva, donde tienen unos mayores incentivos a la colusión (Bain 1951 1956). Paralelamente, también ha de considerarse que el incremento en el nivel de globalización ha potenciado la colaboración empresarial a nivel local, generando una mayor interacción entre estas empresas instaladas (Crouch 2011; Calvo-Mora, Berbel-Pineda, Periáñez \& Suárez 2011).

Además de estas pautas de colaboración, existen otro tipo de barreras de entradas en el sector hotelero. Así, Contractor y Kundu (1998) se refieren a la elevada inversión inicial necesaria en este sector que favorece la instalación de capacidad ociosa. A su vez, este mayor tamaño aumenta la inversión necesaria para alcanzar el tamaño mínimo eficiente (Demsetz 1973; Williamson 1975), lo que reduce la entrada de competidores. Detrás de la búsqueda de esta mejor posición competitiva se encuentran determinados procesos de integración, que favorecen un aumento de las empresas en términos de instalaciones y personal, mejorando su posición de poder frente a sus competidores (Pine \& Qui 2004).

En definitiva, a pesar de que la literatura académica reconoce la importancia del entorno en el emprendimiento hotelero, e incluso se han identificado determinadas barreras de entradas relativas a la colaboración empresarial y disponibilidad de recursos, entre otras, no existe todavía evidencia empírica al respecto. Por lo tanto, este trabajo pretende poner de manifiesto a nivel empírico el papel de las barreras de entrada en la creación de hoteles como forma de dilucidar su potencial efecto sobre la dinámica emprendedora presente en esta industria.

\section{Hipótesis y variables}

A partir de la literatura académica, se puede afirmar que las empresas hoteleras instaladas en una determinada localización pueden colaborar o actuar conjuntamente 
buscando incrementar su rentabilidad. Ejemplos de este tipo de prácticas han sido la búsqueda de un desarrollo conjunto de la imagen local (Andrades-Caladito, SánchezRivero \& Pulido-Fernández 2012), y el establecimiento de una política de precios consensuados (Balaguer \& Pernías 2013). Estos acuerdos estratégicos ocurren con mayor facilidad en entornos donde exista un menor nivel de competencia, lo que significa la presencia de una elevada concentración de mercado (Bain 1951 1956). A su vez, este tipo de prácticas pueden ir encaminadas a reducir la entrada de competidores, como forma de mantener una determinada cuota de mercado entre las empresas ya instaladas (Suzuki 2013; Rodríguez \& Murdy 2006; De la Cruz 2003). En este sentido, para contrastar empíricamente estos hechos se plantea la siguiente hipótesis:

H1: Una mayor concentración de mercado (menor nivel de competencia) reduce la creación empresarial.

La inversión incial necesaria para emprender un negocio depende del tamaño que presenten los competidores (Rosenthal \& Strange 2003). De este modo, en aquellas zonas donde los establecimientos hoteleros presenten un mayor tamaño, la inversión inicial se verá incrementada (Contractor \& Kundu 1998). Este aumento de la inversión inicial necesaria supone una barrera a la entrada de nuevos competidores, de modo que se plantea la siguiente hipótesis:

H2: El volumen de inversión inicial necesario afecta negativamente a la creación empresarial

A partir de la concreción de procesos de integración empresarial, los establecimientos hoteleros pueden afectar no solo a la inversión inicial necesaria en términos de tamaño, sino también al servicio prestado (Pine \& Qui 2004). De este modo, en aquellos destinos donde los establecimientos ofrezcan más servicios, la inversión inicial será mayor, independientemente del tamaño del establecimiento. Teniendo en cuenta esta consideración, se plantea la utilización de una proxy del nivel de servicios ofrecidos a partir del personal contratado, con el objeto de contrastar esta hipótesis.

H3: El volumen de servicios ofrecido afecta negativamente a la creación empresarial

Otro aspecto relevante a incluir en el análisis es el hecho de que la creación empresarial depende de la demanda esperada, de modo que en aquellas localizaciones donde existan mayores oportunidades de negocio se esperar una mayor dinámica emprendedora. Este trabajo tiene en consideración estos factores incluyendo variables relativas a la rentabilidad media obtenida, el nivel de ocupación y el tamaño de mercado. A su vez, y como forma de incluir también en el análisis la existencia de oportunidades de negocio como consecuencia de la desaparición de empresas instaladas, se incorpora el cierre de establecimientos hoteleros.

La Tabla 1 presenta todas las variables de análisis y su relación esperada con el nivel de creación de empresarial, esto es, el número de nuevas empresas hoteleras creadas en cada punto turístico. Este estudio evalúa 8,992 establecimientos hoteleros ubicados en los 97 puntos turísticos que, de acuerdo al Instituto Nacional de Estadística de España, componen la totalidad de la oferta turística nacional. Además, se ha utilizado el criterio de mínima distancia para considerar en el análisis empírico el área de influencia de cada punto turístico. Por tanto, se asume que cada nueva empresa se crea en el área de influencia del punto turístico más cercano. Se han utilizado dos fuentes de información para la construcción de la muestra. Por una parte, la base de datos SABI para la obtención de los datos económicos y financieros propios de cada hotel. Por otra, la Encuesta de Ocupación Hotelera (EOH), que publica mensualmente el Instituto Nacional de Estadística de España (INE), para la obtención de datos relacionados con la actividad del mercado hotelero en España.

Tabla 1 - Variables: definición y signo esperado

\begin{tabular}{|c|c|c|c|c|c|}
\hline Variable & Medida & Unidad de Medida & Cálculo & Fuente & $\begin{array}{l}\text { Relación } \\
\text { esperada }\end{array}$ \\
\hline \multicolumn{6}{|c|}{ Variable endógena } \\
\hline Creación & $\begin{array}{l}\text { № de Empresas } \\
\text { Creadas }\end{array}$ & Unidades & $\begin{array}{l}\text { № de Empresas creadas en el punto } \\
\text { turístico i y en el momento } t\end{array}$ & SABI & \\
\hline \multicolumn{6}{|c|}{ Variables exógenas } \\
\hline Concentración & $\begin{array}{l}\text { Índice de } \\
\text { Herfindahl }\end{array}$ & Índice & $\sum_{j=1}^{J}\left(s^{2}\right)_{j}^{i}$ & SABI & - \\
\hline Rentabilidad & $\begin{array}{l}\text { Rentabilidad } \\
\text { Económica Media }\end{array}$ & $\%$ & 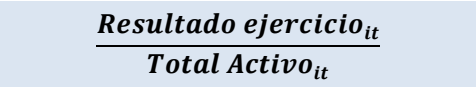 & SABI & + \\
\hline $\begin{array}{l}\text { Nivel de } \\
\text { Ocupación }\end{array}$ & $\begin{array}{c}\text { Tasa de } \\
\text { Ocupación }\end{array}$ & $\%$ & $\frac{\sum_{m=1}^{12} \text { Ocupación media mensual }_{\text {itm }}}{12}$ & INE & + \\
\hline Mercado & Nivel de Ingresos & $\operatorname{Ln}($ Euros $(€))$ & $\operatorname{Ln}\left(\sum_{j=1}^{\mathrm{J}}\right.$ Total Ingresos $\left.{ }_{j i t}\right)$ & SABI & + \\
\hline Inversión & $\begin{array}{l}\text { Tamaño medio } \\
\text { del hotel }\end{array}$ & $\begin{array}{c}\text { Ratio } \\
\text { (Plazas por hotel) }\end{array}$ & $\frac{\text { Número de }^{\text {plazas }}}{\text { Número de establecimientos }_{i t}}$ & INE & - \\
\hline Personal & $\begin{array}{l}\text { Personal Medio } \\
\text { Empleado }\end{array}$ & $\begin{array}{c}\text { Ratio } \\
\text { (Empleados por plaza) }\end{array}$ & $\frac{\text { Número de empleados }_{i t}}{\text { Número de establecimientos }_{i t}}$ & INE & - \\
\hline Cierres & $\begin{array}{l}\text { № de Empresas } \\
\text { Cerradas }\end{array}$ & Unidades & $\begin{array}{l}\text { № de Empresas cerradas en el punto } \\
\text { turístico i y en el momento } \mathrm{t}\end{array}$ & SABI & + \\
\hline
\end{tabular}

Fuente: Autores. Notas: $i$ representa cada punto turístico, $t$ identifica el período de tiempo, $m$ identifica cada mes del año (esta unidad de medida es utilizada cuando la información mensual está disponible), y $j$ representa cada empresa. Finalmente, $s$ es la cuota de mercado de la empresa $j$ en el punto turístico $i$, definida como el cociente entre sus ventas y las ventas totales en ese punto turístico. Cada empresa hotelera ha sido asignada al punto turístico más próximo a su ubicación. Si está presente en varios puntos turísticos a través del establecimiento de delegaciones, las variables relativas al mercado hotelero se han calculado como una media ponderada en función de su tamaño en cada uno de ellos. 
El estudio descriptivo de las variables de análisis se recoge en la Tabla 2. De este modo, se puede comprobar que la creación de empresas ha tenido una tendencia negativa a lo largo del período considerado (2005-2011). Esta reducción se acompaña de una importante caída en la rentabilidad media, en el nivel de ocupación y en el total de ingresos medio, especialmente, en el último trienio. Estos datos podrían ser indicativos de la incidencia de la crisis económica y, en particular, de la caída de demanda asociada a la misma, sobre el emprendimiento hotelero.

Tabla 2 - Análisis descriptivo de las variables utilizadas en el análisis

\begin{tabular}{|c|c|c|c|c|c|c|c|c|c|}
\hline Año & Estadístico & Creación & Concentración & Rentabilidad & Ocupación & Mercado* & Inversión & Personal & Cierres \\
\hline \multirow{2}{*}{2005} & Media & 5.42 & 0.09 & -2.02 & 49.48 & 82,595 & 139.89 & 6.78 & 1.88 \\
\hline & Desv. Std. & 6.97 & 0.07 & 5.49 & 11.54 & 172,282 & 145.41 & 1.58 & 2.76 \\
\hline \multirow{2}{*}{2006} & Media & 4.28 & 0.09 & -2.12 & 50.91 & 94,717 & 144.82 & 6.60 & 2.42 \\
\hline & Desv. Std. & 6.61 & 0.08 & 4.60 & 12.10 & 202,965 & 149.81 & 1.56 & 3.19 \\
\hline \multirow{2}{*}{2007} & Media & 3.30 & 0.10 & -2.10 & 50.61 & 92,398 & 147.85 & 6.67 & 2.24 \\
\hline & Desv. Std. & 5.41 & 0.07 & 6.65 & 12.38 & 198,931 & 152.97 & 1.64 & 2.80 \\
\hline \multirow{2}{*}{2008} & Media & 2.41 & 0.09 & -3.27 & 48.21 & 89,179 & 150.64 & 7.13 & 1.30 \\
\hline & Desv. Std. & 3.75 & 0.07 & 6.57 & 12.09 & 189,356 & 157.13 & 1.72 & 2.44 \\
\hline \multirow{2}{*}{2009} & Media & 2.11 & 0.08 & -5.72 & 45.17 & 81,033 & 152.07 & 7.69 & 2.26 \\
\hline & Desv. Std. & 3.42 & 0.06 & 5.44 & 11.30 & 164,618 & 158.84 & 1.82 & 4.42 \\
\hline \multirow{2}{*}{2010} & Media & 1.81 & 0.09 & -5.55 & 46.28 & 81,118 & 154.38 & 7.80 & 2.78 \\
\hline & Desv. Std. & 3.07 & 0.06 & 6.35 & 11.86 & 169,394 & 157.93 & 1.96 & 4.60 \\
\hline \multirow{2}{*}{2011} & Media & 0.90 & 0.10 & -5.74 & 47.72 & 70,180 & 157.22 & 7.79 & 3.19 \\
\hline & Desv. Std. & 1.55 & 0.07 & 5.90 & 13.83 & 143,959 & 157.96 & 1.97 & 4.34 \\
\hline \multirow{2}{*}{ Total } & Media & 2.89 & 0.09 & -3.79 & 48.36 & 84,460 & 149.46 & 7.20 & 2.29 \\
\hline & Desv. Std. & 4.96 & 0.07 & 6.10 & 12.28 & 177,793 & 153.70 & 1.82 & 3.64 \\
\hline
\end{tabular}

Fuente: Autores. Notas: Desv. Std. Significa desviación estándar. * Datos sin transformación logarítmica.

Cabe también destacar la pervivencia en el mercado de las grandes empresas, manifestada a través del incremento en el tamaño medio de las empresas establecidas en el mercado. Esto puede deberse a dos causas: la mayor eficiencia que presentan las grandes empresas (Demsetz 1973), o las mayores barreras de salida que presentan los establecimientos hoteleros de mayor tamaño y que provocan que sea más difícil desinvertir sus activos.

En relación con los empleados, el incremento en el número medio en los últimos años parece derivarse del alza protagonizada por los servicios ofrecidos por el sector, siendo las empresas con menos trabajadores las que abandonan el mercado. Sin embargo, no debe asumirse que esto sea una consecuencia de la desaparición de las empresas de menor tamaño por otros motivos, como podrían ser el hecho de no alcanzar un tamaño mínimo eficiente $o$ las mayores dificultades para obtener financiación en época de desaceleración económica.

Finalmente, debe indicarse que el cierre de empresas presenta sus mayores valores en los dos últimos años (2010 y 2011), lo cual, al no ir acompañado de un incremento en la creación, pone de manifiesto la destrucción de tejido productivo sectorial. Este aumento en el cierre de hoteles no genera un incremento significativo del nivel de concentración, lo que, junto con el aumento del tamaño medio de los establecimientos hoteleros antes comentado, revela un incremento en la intensidad competitiva y una disminución en la cuota de mercado de las empresas instaladas. De hecho, este reparto de la cuota de mercado parece deberse a dos efectos: 0 bien a un reparto a favor de empresas instaladas de menor cuota, o bien a un reparto a favor de las nuevas empresas que entran en el mercado, aspecto que reduciría la cuota de mercado de las empresas de mayor tamaño.

\section{Estudio empírico}

Este trabajo analizar la creación de empresarial en el sector hotelero español utilizando como variable dependiente el número de empresas creadas en cada punto turístico y momento del tiempo. Por tanto, dada la naturaleza del fenómeno a estudiar (número de empresas creadas), y de acuerdo con literatura previa, una metodología empírica apropiada es el modelo de Poisson (Baltagi 2010). No obstante, este tipo de estimación no está exenta de crítica debido a que asume que la media es igual a la varianza en el proceso analizado. Por este motivo, se ha planteado como alternativa la estimación de un modelo Binomial Negativa, donde se generaliza el modelo de Poisson introduciendo un efecto inobservado $\phi$ igual a la media condicional y asumiendo que $\exp (\phi)$ sigue una distribución gamma con esperanza unitaria y varianza $\alpha$. El test de sobredispersión muestra que no es posible aceptar la hipótesis de equidispersión, de modo que se plantea la estimación de un modelo binomial negativo.

Una vez determinado que el modelo binomial negativo mejora la estimación, cabe señalar que una característica de ambos modelos, Poisson y Binomial Negativa, es el hecho de que, en el caso de nuestro objeto de estudio, asumen que los factores que determinan una mayor o menor creación empresarial se identifican con los factores que determinan la inexistencia de creación empresarial. Sin embargo, debe reconocerse que en el sector hotelero existen factores como prohibiciones, ausencia de licencias, limitaciones de espacio físico, entre otros, que podrían impedir específicamente la creación empresarial. Por este motivo, este trabajo ha aplicado una tercera metodología que es la combinación de modelo truncado, en el cual se consideran exclusivamente aquellas observaciones donde existe creación empresarial, y otro logístico, que incorpora aquellas donde no existe.

Los resultados de las estimaciones realizadas se encuentran en la Tabla 3. Así, cabe destacar que el incremento de un punto en el nivel de concentración de mercado (concentración) supone una reducción de entre el $0.82 \%$ y el $0.91 \%$ en el número de empresas creadas. A su vez, destaca la sensibilidad de la creación empresarial respecto 
al volumen de ingresos (mercado), de modo que los resultados obtenidos sugieren que un incremento de un $1 \%$ en el volumen de ingresos totales supone un incremento en la creación empresarial de entre un 36.8\% y un $66.1 \%$. En relación con el resto de variables significativas, se observa que un mayor nivel de rentabilidad incrementa el número de nuevas empresas, al igual que el nivel de ocupación que también se encuentra, relacionado positivamente con la intensidad de entrada de competidores. Por su parte, el nivel de inversión afecta negativamente al número de empresas creadas, lo que se vincula con la necesidad de un mayor desembolso de recursos inicial. En esta misma línea se sitúa el nivel de servicios ofrecido por las empresas hoteleras que actúan en el mercado, relacionado negativamente con la creación empresarial. Además, en todas las estimaciones, las variables utilizadas presentan una elevada significatividad, mostrando la robustez del modelo estimado. En relación con el modelo truncado estimado para los casos en los cuales no existe creación empresarial, los resultados muestran que un bajo tamaño del mercado y un elevado tamaño medio de las empresas instaladas son características significativas que impiden la entrada de nuevos competidores. Ahora bien, esta interpretación está condicionada o limitada por la inexistencia de un número de observaciones elevado.

Tabla 3 - Modelos empíricos sobre emprendimiento en el sector hotelero

\begin{tabular}{|c|c|c|c|c|c|c|c|c|}
\hline \multirow{3}{*}{ Variables } & \multicolumn{8}{|c|}{ Variable dependiente: número de empresas creadas en cada punto turístico } \\
\hline & \multicolumn{2}{|c|}{$\begin{array}{l}\text { Modelo } \\
\text { Poisson }\end{array}$} & \multicolumn{2}{|c|}{$\begin{array}{c}\text { Modelo } \\
\text { Binomial Negativa }\end{array}$} & \multicolumn{2}{|c|}{$\begin{array}{c}\text { Modelo Truncado } \\
\text { Sí existe creación } \\
\text { empresarial }\end{array}$} & \multicolumn{2}{|c|}{$\begin{array}{c}\text { Modelo Logístico } \\
\text { No existe creación } \\
\text { empresarial }\end{array}$} \\
\hline & Coef & Imp & Coef & Imp & Coef & $\operatorname{Imp}$ & Coef & Odds \\
\hline Concentración & $-1.717^{* * *}$ & 0.180 & $-2.198^{* * *}$ & 0.111 & $-2.412^{* *}$ & 0.090 & 2.525 & 12.487 \\
\hline Rentabilidad & $0.014^{* * *}$ & 1.014 & $0.011^{*}$ & 1.011 & 0.010 & 1.010 & -0.020 & 0.980 \\
\hline Ocupación & $0.011^{* * *}$ & 1.011 & $0.014^{* *}$ & 1.014 & $0.013^{*}$ & 1.013 & -0.022 & 0.978 \\
\hline Mercado & $0.507^{* * *}$ & 1.661 & $0.358^{* * *}$ & 1.430 & $0.313^{* * *}$ & 1.368 & $-0.643^{* * *}$ & 0.526 \\
\hline Inversión & $-0.003^{* * *}$ & 0.997 & $-0.003^{* * *}$ & 0.997 & $-0.002^{* * *}$ & 0.998 & $0.005^{* * *}$ & 1.005 \\
\hline Personal & $-0.074^{* * *}$ & 0.929 & $-0.081^{* * *}$ & 0.923 & $-0.122^{* * *}$ & 0.885 & 0.015 & 1.015 \\
\hline Cierres & $0.018^{* * *}$ & 1.018 & $0.048^{* * *}$ & 1.049 & $0.057 * * *$ & 1.058 & -0.001 & 0.999 \\
\hline Constante & $-3.929 * * *$ & 0.020 & $-2.544^{* * *}$ & 0.079 & $-1.837^{* *}$ & 0.159 & 5.334 & 207.187 \\
\hline Alfa & & & -0.961 & 0.383 & 0.381 & & & \\
\hline Obsv. & \multicolumn{2}{|c|}{666} & \multicolumn{2}{|c|}{666} & \multicolumn{2}{|c|}{499} & \multicolumn{2}{|c|}{666} \\
\hline Pseudo-R ${ }^{2}$ & \multicolumn{2}{|c|}{0.3812} & \multicolumn{2}{|c|}{0.1359} & \multicolumn{2}{|c|}{0.1279} & \multicolumn{2}{|c|}{0.1219} \\
\hline $\begin{array}{l}\text { Log } \\
\text { Likelihood }\end{array}$ & \multicolumn{2}{|c|}{-1401.824} & \multicolumn{2}{|c|}{-1279.110} & \multicolumn{2}{|c|}{-946.69} & \multicolumn{2}{|c|}{-329.325} \\
\hline $\begin{array}{l}\text { LR test } \\
(\mathrm{alfa}=0)\end{array}$ & & & \multicolumn{2}{|c|}{$245.43^{* * *}$} & & & & \\
\hline LR test $\left(\chi^{2}\right)$ & & & & & 277. & & & \\
\hline FIV & \multicolumn{6}{|c|}{2.28} & \multicolumn{2}{|c|}{2.28} \\
\hline
\end{tabular}

Fuente: Autores. Notas: Coef. representa los coeficientes, Imp. el ratio de incidencia que indica en qué medida un cambio en la variable dependiente afecta a la variable explicada, y Odds es la razón de momios indicativa de en qué medida es posible que una condición se presente en el grupo de análisis frente a la posibilidad de que se presente en el grupo base o de control (quebrada vs. activa). Pseudo- $R^{2}$ es una medida de la bondad de ajuste del modelo. Log likelihood es el valor de la función de máxima-verosimilitud. LR test ( $a l f a=0$ ) es un test de sobredispersión bajo la hipótesis nula de que el parámetro de dispersión alfa es igual a cero. LR test $(\chi 2)$ es un test que contrasta la bondad del ajuste, asintóticamente distribuido como una $\chi 2$, bajo la hipótesis nula de no significación conjunta de los coeficientes de las variables explicativas. FIV es el factor de incremento de la varianza, que refleja el grado de multicolinealidad entre las variables explicativas. ${ }^{* * * * * * *}$ significativo al $1 \%, 5 \%$ y $10 \%$ respectivamente.

\section{Conclusiones}

La importancia del emprendimiento como elemento impulsor y dinamizador del crecimiento económico ha sido destacada en la literatura académica siendo objeto de diferentes investigaciones empíricas. La mayoría de trabajos se ha focalizado en el estudio de las características del emprendedor, así como en el contexto corporativo que rodea el proceso emprendedor. Por tanto, existen otros aspectos todavía no analizados como, por ejemplo, la incidencia de determinadas características sectoriales sobre la dinámica emprendedora.

En este sentido, este trabajo se ha centrado en identificar y cuantificar el impacto de las barreras de entrada existentes en una localización sobre la intensidad emprendedora en el sector hotelero español durante el período 2005-2011.

Tras una revisión de la literatura donde se han identificado los principales factores que pueden afectar al proceso emprendedor, los resultados obtenidos en esta investigación destacan la importancia de la consideración del entorno sectorial en la creación empresarial. En concreto, se observa, en línea con el planteamiento teórico de estudios anteriores, que el nivel de concentración de mercado puede favorecer la aparición de prácticas encaminadas a reducir la entrada de competidores. Asimismo, otro aspecto destacado se relaciona con la identificación del impacto que una mayor inversión ejerce sobre la entrada de competidores. De hecho, algunos estudios previos apuntan la potencial existencia de prácticas disuasorias a la entrada mediante la instalación de capacidad ociosa, si bien la baja liquidez de la inversión en el sector hotelero puede estar detrás de su efecto sobre la reducción en la entrada de competidores y no necesariamente deberse a la existencia de prácticas anticompetitivas.

En relación con el efecto de los procesos de integración experimentados en el sector durante los últimos años, los resultados obtenidos para las variables vinculadas a la 
inversión inicial y al nivel de servicios ofrecidos, indican que estos procesos pueden actuar como un elemento reductor de la entrada de nuevos competidores. Así, obtenemos que es conveniente incluir en el análisis de los procesos de integración sectorial no solo aspectos relativos al nivel de eficiencia sino también a su potencial efecto sobre la entrada de competidores, lo que en el medio plazo puede suponer una reducción del nivel de competencia que puede afectar al nivel de precios.

Si bien no era el principal foco principal de este trabajo, los resultados también parecen señalar que la creación empresarial no depende en gran medida del nivel de rentabilidad obtenida en el mercado, sino que se ve más afectada por los niveles de ocupación y, especialmente, por el tamaño de aquel. De hecho, las estrategias de especialización y la existencia de una mayor variedad de la oferta en aquellos puntos turísticos de mayor afluencia turística, pueden explicar esta mayor creación empresarial.

A pesar de la importancia de esta industria en la economía española y su posición relevante en el entorno turístico mundial, todavía no existen estudios empíricos de esta naturaleza. Por tanto, este trabajo aporta la primera evidencia empírica en el ámbito académico relativa a la importancia de la consideración de las barreras de entrada en el sector hotelero. Además, sus resultados pueden ser utilizados para el análisis y diseño de políticas de eliminación de barreras de entrada que favorezcan un incremento en el nivel de competencia encaminado a generar mayores niveles de eficiencia. De este modo, se plantean como potenciales líneas futuras de investigación el análisis del impacto que ejercen medidas anticompetitivas sobre el nivel de emprendimiento hotelero y, por extensión, a su desarrollo económico.

\section{Referencias}

Acs, Z. J., Braunerhjelm, P., Audretsch, D.B. \& Carlsson, B. (2009). The knowledge spillover theory of entrepreneurship. Small Business Economics, 32(1), 15-30.

Andrades-Caladito, L., Sánchez-Rivero, M. \& Pulido-Fernández, J.I. (2012). Differentiating competitiveness through tourism image assessment: an application to Andalusia (Spain). Journal of Travel Research, 20(10), 1-14.

Ateljevic, J. \& Page, S.J. (2009). Tourism and entrepreneurship. International perspective. UK: Elsevier.

Bain, J. S. (1951). Relation of profit rate to industry concentration: American manufacturing, 1936-1940. Quarterly Journal of Economics, 65(3), 293-324.

Bain, J. S. (1956). Barriers to new competition. Cambridge: Harvard University Press.

Balaguer, J. \& Pernías, J.C. (2013). Relation between spatial agglomeration and hotel prices. Evidence from business and tourism consumers. Tourism Management, 36(3), 391-400.

Baltagi, B. (2010). Econometric Analysis of Panel Data. London: JohnWiley \& Sons.

Calvo-Mora, A., Berbel-Pineda, J.M., Periáñez, R. \& Suárez, E.M. (2011). Determining factors of a city's tourism attractiveness. Tourism \& Management Studies, 7(1), 9-23.

Carlsson, B., Braunerhjelm, P., McKelvey, M., Olofsson, C., Persson, L. \& Ylinenpa, H. (2013). The evolving domain of entrepreneurship research. Small Business Economics, 41(4), 913-930,

Contractor, J.F. \& Kundu, K.S. (1998). Modal choice in the world of alliances: analyzing organizational forms in the international hotel sector. Journal of International Business Studies, 29(2), 325- 58.
Crouch, G. I. (2011). Destination competitiveness: an analysis of determinant attributes. Journal of Travel Research, 50(1), 27-45.

Santos, Margarida Custódio, Ferreira, Ana Maria \& Costa, Carlos (2014). Influential factors in the competitiveness of mature tourism destinations. Tourism \& Management Studies, 10(1), 73-81.

De La Cruz T. (2003). Developers overcome obstacles for desirable projects. Hotel \& Motel Management, 218(14), 26-37.

Demsetz, H. (1973). Industry structure, market rivalry and public policy. Journal of Law and Economics, 16(1), 1-9.

Figueroa-Armijos, M., Dabson, B. \& Johnson, T.G. (2012). Rural entrepreneurship in a time of recession. Entrepreneurship Research Journal, 2(1), 10-27.

Gémar-Castillo, G. \& Jiménez-Quintero, J.A. (2013). Retos estratégicos de la industria hotelera española del siglo XXI: Horizonte 2020 en países emergentes. Tourism \& Management Studies, 9(2), 13-20.

Jaafar, M., Abdul-Aziz, A.R., Maideen, S.A., Mohd, S.Z. (2011). Entrepreneurship in the tourism industry: Issues in developing countries. International Journal of Hospitality Management, 30(1), 827-835.

Justo, R., y Díaz, C. (2012). Incidencia de los modelos de referencia en la creación de empresas. Efectos mediadores y de género. Economía Industrial, 383(1), 111-123.

Kalnins, A. \& Chung, W. (2004). Resource-seeking agglomeration: A study of market entry in the lodging industry. Strategic Management Journal, 25(1), 689-699.

Li, L. (2008). A review of entrepreneurship research published in the hospitality and tourism management journals. Tourism Management, 29(1), 1013-1022

Li, L., Ching-Yick, R. \& Zhao, T. (2009). An empirical study of corporate entrepreneurship in hospitality companies. International Journal of Hospitality \& Tourism Administration, 10(3), 213-231.

Martínez-López, A. M. \& Vargas-Sánchez, A. (2013). Factores con un especial impacto en el nivel de innovación del sector hotelero español. Tourism \& Management Studies, 9(2), 7-12.

Monterrubio, J.C. \& Mendoza-Ontiveros, M.M. (2014). Tourism and the demonstration effect: empirical evidence. Tourism \& Management Studies, 10(1), 97-103.

Mottiar, Z. \& Ryan, T. (2007). The role of SMEs in tourism development: an industrial district approach applied to Killarner, Ireland. In R. Thomas \& M. Augustyn (eds.), Tourism in the New Europe: Perspectivas on SME policies and practices (pp. 63-78). Amsterdam: Elsevier.

Parwada, J.T. (2008). The genesis of home bias? The location and portfolio choices of investment company start-ups. Journal of Financial and Quantitative Analysis, 43(1), 245-266.

Pine, R \& Qui, P. (2004). Barriers to hotel chain development in China. International Journal of Contemporary Hospitality Management, 16(1), 37-44.

Porter, M. (2008). The five competitive forces that shape strategy. Harvard Business Review, 1(1), 23-40.

Ramos-Rodríguez, A.R., Medina-Garrido, J.A. \& Ruiz-Navarro, J. (2012). Determinants of hotels and restaurants entrepreneurship: a study using GEM data. International Journal of Hospitality Management, 31(1), 579-587.

Rodríguez, A.E. \& Murdy, J. (2006). Anti-competitive practices in the tourism industry: the case of small economies. Journal of Business \& Economics Research, 4(10), 43-49.

Russell, R. \& Faulkner, B. (2004). Entrepreneurship, chaos and the tourism area lifecycle. Annals of Tourism Research, 31(3), 556-579.

Rosenthal, S. \& Strange, W. (2003). Geography, industrial organization, and agglomeration. Review of Economics and Statistics, 85(2), 377-393.

Sánchez, J.C., Lanero, A. \& Yurrebaso, A. (2005). Variables determinantes de la intención emprendedora en el contexto universitario. Revista de Psicología Social Aplicada, 15(1-2), 37-60.

Santos-Góes, A. O. \& Vianna-Brugni, T. (2014). Entrepreneurship from the perspective of small business support entities. Tourism \& Management Studies, 10(Special Edition), 19-25.

Stam, E. (2007). Why butterflies don't leave: locational behavior of entrepreneurial firms. Economic Geography, 83(1), 27-50. 
Stenholm P., Acs, Z. \& Wuebker, R. (2013) Exploring country level institutional arrangements on the rate and type of entrepreneurial activity. Journal of Business Venturing, 28(1), 176-193.

Stewart, S., Wayne, H. Jr. \& Roth, P.L. (2001). Risk propensity differences between entrepreneurs and managers: A meta-analytic review. Journal of Applied Psychology, 86(1), 145-153.

Suzuki, J. (2013). Land use regulation as a barrier to entry: evidence from the Texas lodging industry. International Economic Review, 54 (2), 495-593.

Thurik, A.R. \& Dejardin, M. (2011). The impact of culture on entrepreneurship. The European Business Review, 1(2), 57-59.

Verheul, I., Wennekers, S., Audretsch, D. \& Thurik, A. R. (2002). Entrepreneurship: determinants and policy in a European-US comparison. Economics of Science, Technology and Innovation, 27(1), 11-81.

Williamson, 0. (1975). Markets and hierarchies: analysis and antitrust implications. New York: Free Press.

Recibido: 15.05.2014

Aceptado: 15.11.2014 